\title{
The Expression And Significance Of RIPK3 In Cutaneous Lichen Planus And The Epidemiological Analysis
}

\author{
Mengdi Zhang, Min Wang, Qiqi Duan and Yan Zheng* \\ Department of Dermatology, the Second Affiliated Hospital, School of Medicine, Xi'an Jiaotong University, China
}

\begin{abstract}
Received: December 27,2018; Accepted: January 17, 2019 ; Published: January 21, 2019
*Corresponding author: Yan Zheng, Department of Dermatology, the Second Affiliated Hospital, School of Medicine, Xi'an Jiaotong University, 157 Xiwu Road, Xi'an 710004, China, Tel: 15319931238,E-mail: zenyan66@126.com
\end{abstract}

\begin{abstract}
Objectives: RIPK3 is a member of the receptor-interacting protein kinase family. The RIPK3 protein is a TNFR1-regulated transcription factor that plays an important role in cell stress caused by various factors such as pathogen infection, inflammation, cell differentiation and cell damage. When cells are exposed to external stimuli, RIPK3 activates the downstream transcription factors to cause a series of reactions that ultimately lead to cell survival or death. However, the roles of RIPK3 in lichen planus has not been reported. In this study we compared the expression of RIPK3 protein in normal skin tissue and cutaneous lichen planus lesion, and analyzed the epidemiology characteristics of the patients. It is speculated that RIPK3 may play a role in the development of cutaneous lichen planus.
\end{abstract}

Methods: The SP method of immunohistochemistry was carried out to detect the expression of RIPK3 protein in 30cases of normal skin tissues and 50 cases of cutaneous lichen planus lesions. Data analysis software was used to analyze the epidemiology characteristics.

Results: In 1854 patients from 2010 to 2018 in our hospital, the peak age of the patients diagnosed was 30-70 years old. There was no significant difference in sex ratio, but the mean age of diagnosis of male and female patients was statistically different. In normal skin tissues, RIPK3 protein was weakly expressed in the basal layer of the skin and in the bottom of the spinous layer, and it was mainly expressed in the nucleus. In the cutaneous lichen planus, RIPK3 protein was strongly expressed in the spinous layer, basal layer and part of the granular layer, and it was mainly expressed in the cytoplasm. The positive rate of the expression of RIPK3 protein in cutaneous lichen planus lesions was higher than that in normal skin tissues.

Conclusions: The age of onset of males is earlier than that of females in the pathogenesis of cutaneous lichen planus, but there is no difference in sex ratios. The high incidence age of males and females is in the middle and old stage. The transfer of RIPK3 protein as a transcription factor from the nucleus to the cytoplasm in cutaneous lichen planus may play a regulatory role in the pathogenesis of cutaneous lichen planus, which provides a significant thought and method for study of the pathogenesis of cutaneous lichen planus.

Keywords: Cutaneous lichen planus; skewed distribution; RIPK3; Transcription factor;

\section{Introduction}

Cutaneous lichen planus (LP) is an idiopathic inflammatory skin disease with an incidence of less than $0.1 \%$ [1], and the malignant transformation is infrequent [2]. However, It can make up $0.4 \%$ to $1.2 \%$ of all dermatology referrals and features [3]. Cutaneous LP typically presents as a papulosquamous eruption with flat-topped, violaceous, papular lesions of varying size, often described using the 'six P's' (purple, pruritic, polygonal, planar, papules, and plaques) and characterized by the classic Wickham striae [4-7]. Although LP has variable morphologic manifestation, it has a consistent histological phenotype[8]. The most frequently involved areas are skin and oral mucosa are. In addition, scalp hair, nails, genitalia, esophagus, and conjunctiva can also be affected. One or several areas can be involved concomitantly or sequentially [1].

Receptor-interacting protein kinase 3 (RIPK3) is a member of the receptor-interacting protein kinase family of serine/ threonine protein kinases. The human RIPK3 gene is encoded on chromosome 14q11. The RIPK3 protein has a molecular weight of $53 \mathrm{kDa}$ and encodes 518 amino acids. It contains a kinaseactive N-terminal kinase domain and a C-terminal RHIM domain that binds to multiple proteins [9].RIPK3 is highly expressed in lymphocytes, monocytes and NK cells, and is so expressed in liver cells, muscle cells, retinal cells, and epithelial cells [10-15]. RIPK3 is generally considered to be a key driver of necroptosis, which is a form of non-apoptotic programmed cell death. RIPK3 can rupture cell membranes by inducing programmed cell death to release intracellular compounds that activate immune function and promote inflammation [16-18]. In another case, RIPK3 can also activate downstream factors to promote inflammation independent of necroptosis [19-21].

In this study, we used immunohistochemistry to detect the expression level and expression localization of RIPK3 in cutaneous LP and perform statistical analysis. To explore the mechanism of RIPK3 in the development of cutaneous LP. 


\section{Methods and Materials}

\section{Patients and controls}

The patient sample was achieved from the tissue bank of Department of Dermatology, the Second Affiliated Hospital of Xi'an Jiao tong University. A total of 1854cases of cutaneous LP patients from year2010 to 2018 were collected for analysis, including 965 males and 889 females. 80 patients were randomly selected for immunehisto chemistry, including 30 normal skin tissues achieved from cosmetic surgery (18 males, 12 females, aged 1568 years, mean age 37.7 years), and 50 cutaneous LP tissues (26 males, 24 women, aged 13-66 years, mean age 39.58 years). All samples were obtained with informed consent, approved by the Ethics Committee of the Second Affiliated Hospital of Xi'an Jiao tong University and confirmed by pathological morphological examination.

\section{Immunohistochemistry staining}

4um thick tissue wax pieces were baked, dew axed, and hydrated routinely and incubated with $3 \% \mathrm{H} 2 \mathrm{O} 2$ for 10 min at room temperature to inactivation of endogenous peroxidase. Then the wax pieces were heated under high-pressure for $2 \mathrm{~min}$ in $0.01 \mathrm{M}$ sodium citrate buffer solution ( $\mathrm{pH6}$.0) to retrieval the antigen. Subsequently, $10 \%$ goat serum was used to block the antigen at $37^{\circ} \mathrm{C}$. After 20 minutes, remove the blocking solution without washing. The primary antibody solution of RIPK3 (Abcam, UK, ab56164. Diluted to 1:100) was added drop wise to incubate at $4^{\circ} \mathrm{C}$ overnight, and the negative control was citrate buffer. The secondary antibody was added to incubate at $37^{\circ} \mathrm{C}$ for $30 \mathrm{~min}$. Then, the horseradish peroxidase-labeled streptavidin was incubated at $37^{\circ} \mathrm{C}$ for $20 \mathrm{~min}$. Finally, the DAB coloring solution (Beijing Zhongshang Jinqiao Biotechnology Co., Ltd.) was added for coloration for 3-5 min. We observed the sections under the microscope. We rinsed the sections with clean water, then counterstained the sections with hematoxylin, and then dehydrated, transparentize, dried, and mounted.

\section{Experimental result criterion}

The staining results were evaluated under the microscope by two independent and experienced pathologists and quantified according to the following scoring system: The positive expression was yellow, brownish yellow or brown granules in the cytoplasm and/or nucleus. The staining intensity is divided into 4 grades according to the color of the granules, which is 0 point for no coloring, 1 point for light yellow, 2 points for brownish yellow, and 3 points for brown. The positive rate score was assigned according to the percentage of positive cells $(\leq 5 \%, 0$ points; $6 \% \sim 25 \%, 1$ point; $26 \% \sim 50 \%, 2$ points; $51 \% \sim 75 \%, 3$ points; > $75 \%, 4$ points). Five fields of view were randomly selected under a 400-fold microscope, and 100 tumor cells were counted for each field of view. The total score for each microscope field is the product of two scores, and the average score for the five fields is the final score for RIPK3 expression for each slide. The total scoring criteria were: 0 for negative (-); $1-4$ for weak positive $(+)$; 5-8 for moderate positive $(++)$; 9-12 for strong positive $(+++)$.

\section{Statistical analysis}

Statistical analysis was performed using GraphPad Prism 7.0 (La Jolla, CA, USA) and the SPSS 23.0 software (SPSS Inc, Chicago, IL). Data were expressed as mean \pm SEM. Student's t-test or X2test was used for Statistical comparisons. The Spearman's rank test was used in correlation analyses. $\mathrm{P}<0.05$ was considered statistically significant.

\section{Results}

\section{Cutaneous lichen planus has different incidences in different genders}

The number and mean age of patients diagnosed in our hospital from 2010 to 2018are shown in Table 1 . The number of diagnosed patients showed an upward trend by year, and the spearman correlation coefficient was $0.867, \mathrm{P}<0.05$, which indicates that the number of patients is positively correlated

Table1: Number and mean age distribution of patients with cutaneous lichen planus for year.

\begin{tabular}{|c|c|c|c|c|c|c|c|}
\hline Year & \multicolumn{3}{|c|}{ No. of patients } & Growth rate (\%) & \multicolumn{3}{c|}{ Mean age } \\
\hline & Male & Female & Total & & Male & Female & Total \\
\hline 2010 & 40 & 37 & 77 & & $40.15 \pm 16.08$ & $41.08 \pm 15.31$ & $40.60 \pm 15.83$ \\
\hline 2011 & 65 & 67 & 132 & 71 & $39.71 \pm 18.26$ & $44.79 \pm 17.25$ & $42.29 \pm 18.00$ \\
\hline 2012 & 98 & 99 & 197 & 49 & $41.47 \pm 18.36$ & $45.94 \pm 14.06$ & $43.72 \pm 16.53$ \\
\hline 2013 & 115 & 121 & 236 & 20 & $42.81 \pm 16.70$ & $45.79 \pm 15.94$ & $44.33 \pm 16.39$ \\
\hline 2014 & 93 & 101 & 194 & -18 & $43.46 \pm 15.40$ & $44.42 \pm 16.72$ & $43.96 \pm 16.07$ \\
\hline 2015 & 106 & 94 & 200 & 3 & $42.97 \pm 16.77$ & $48.96 \pm 15.67$ & $45.79 \pm 16.49$ \\
\hline 2016 & 123 & 104 & 227 & 14 & $42.41 \pm 18.35$ & $47.31 \pm 16.07$ & $44.65 \pm 17.47$ \\
\hline 2017 & 165 & 122 & 287 & 26 & $42.29 \pm 16.97$ & $45.43 \pm 15.76$ & $43.63 \pm 16.51$ \\
\hline 2018 & 160 & 144 & 304 & 6 & $43.28 \pm 17.59$ & $46.45 \pm 15.34$ & $44.78 \pm 16.61$ \\
\hline Total & 965 & 889 & 1854 & & $42.37 \pm 16.67$ & $45.95 \pm 15.81$ & $44.09 \pm 16.67$ \\
\hline
\end{tabular}


with the year (Figure 1). The age of the patients diagnosed was relatively large, ranging from a minimum of 3-year old to a maximum of 84-year old. The peak age was 30-70 years old, accounting for $72.92 \%$ of the total number of diagnoses (Figure 2 ). The total age of diagnosis was right skewed distribution, and the skewness coefficient was - 0.066 (Figure 3 ). The overall mean age of diagnoses was $44.09 \pm 16.67$ years old, and there was no statistically significant change in mean age by year (Figure $4, \mathrm{~F}$ $=1.020, \mathrm{P}=0.0419>0.05$ ). The distribution of age and sex is shown in Table 2 and Figure 5. Males accounted for $52.10 \%$ of the total number, and women accounted for $47.90 \%$. There was no significant difference in sex ratio $(P=0.082>0.05)$. As shown in Figure 6-1 and Figure 6-2, the male patients had a left skewed distribution (skewness=0.055), and the female patients had a right skewed distribution (skewness $=-0.171$ ). The mean age of diagnosed male patients was $42.37 \pm 16.67$ years old, and that of female patients were $45.95 \pm 15.81$ years old. The mean age of diagnosis of male and female patients was statistically different $(\mathrm{F}=21.529, \mathrm{P}<0.0001)$.

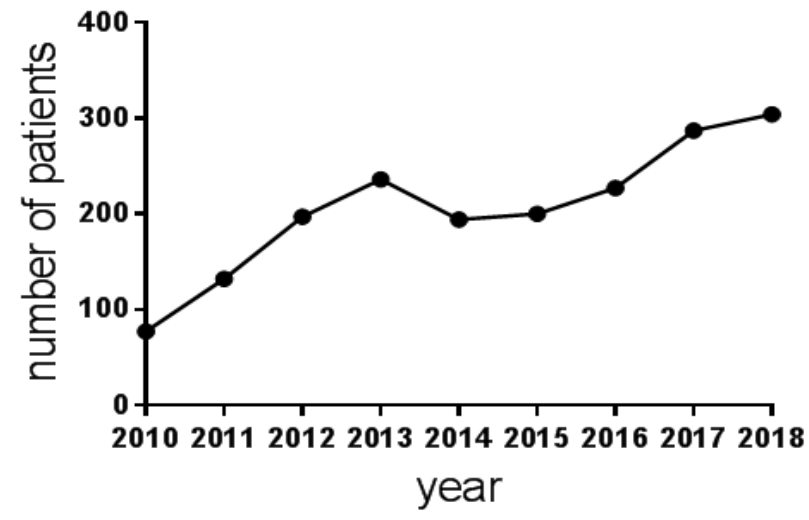

Figure 1: The number of diagnosed patients with cutaneous lichen planus for year $(r=0.867, \mathrm{P}<0.05)$

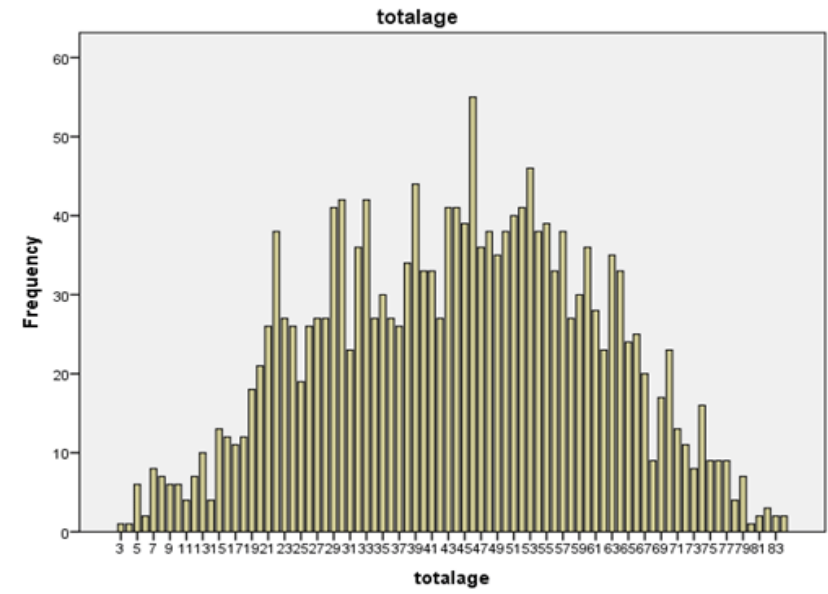

Figure 2: Age frequency distribution of patients with cutaneous lichen planus.

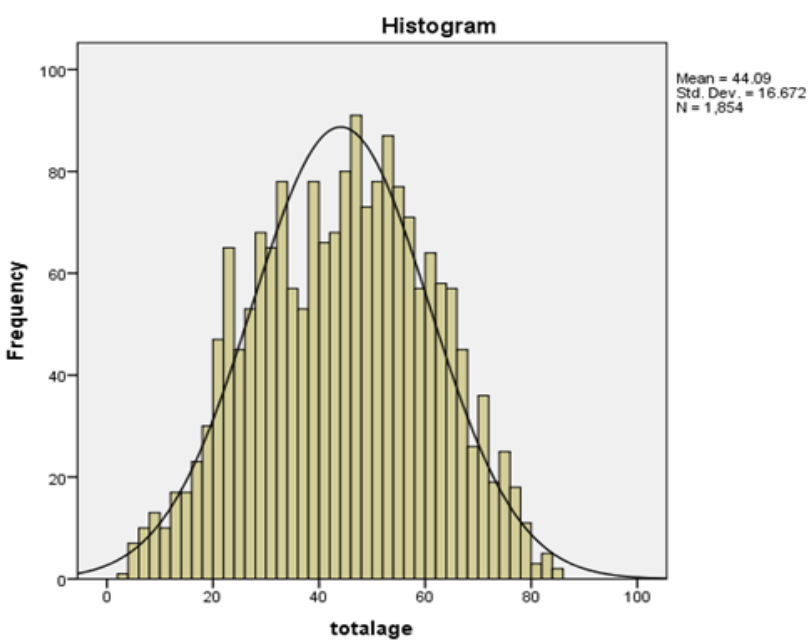

Figure 3: Age skewed distribution of total patients with cutaneous lichen planus (skewness $=-0.066$ ).

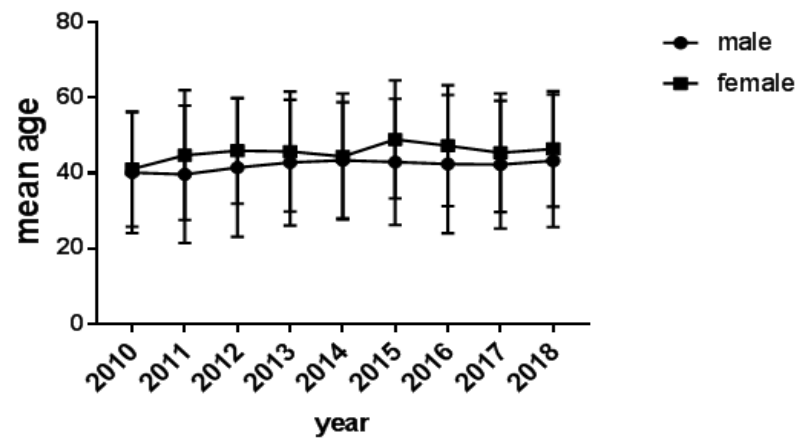

Figure 4: Mean age tendency for year of males and females with cutaneous lichen planus $(\mathrm{F}=1.020, \mathrm{P}=0.0419>0.05)$.

Table2: Age and sex incidence of patients with cutaneous lichen planus.

\begin{tabular}{|c|c|c|c|c|}
\hline Age in year & \multicolumn{3}{|c|}{ No. of patients } & Percentage (\%) \\
\hline & Male & Female & Total & \\
\hline $0-20$ & 92 & 57 & 149 & 8.04 \\
\hline $21-40$ & 362 & 259 & 621 & 33.49 \\
\hline $41-60$ & 347 & 404 & 751 & 40.51 \\
\hline $61-80$ & 158 & 166 & 324 & 17.47 \\
\hline $80+$ & 6 & 3 & 9 & 0.49 \\
\hline Total & 965 & 889 & 1854 & 100 \\
\hline Percentage (\%) & 52.1 & 47.9 & 100 & \\
\hline
\end{tabular}




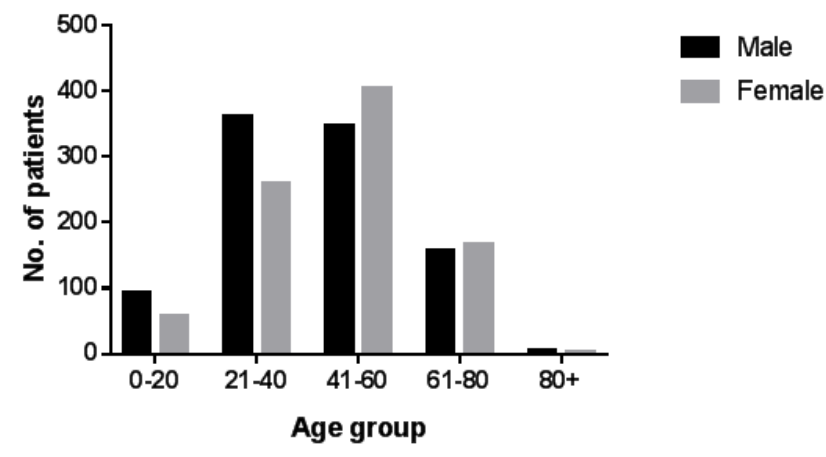

Figure 5: Age and sex distribution of patients with cutaneous lichen planus

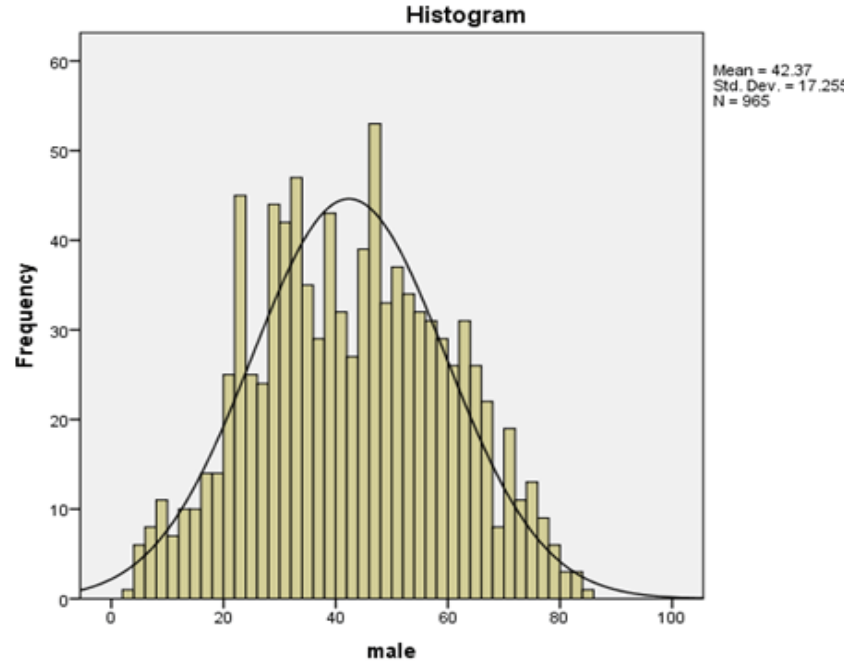

Figure 6.1: Age skewed distribution of males with cutaneous lichen planus (skewness $=0.055$ ).

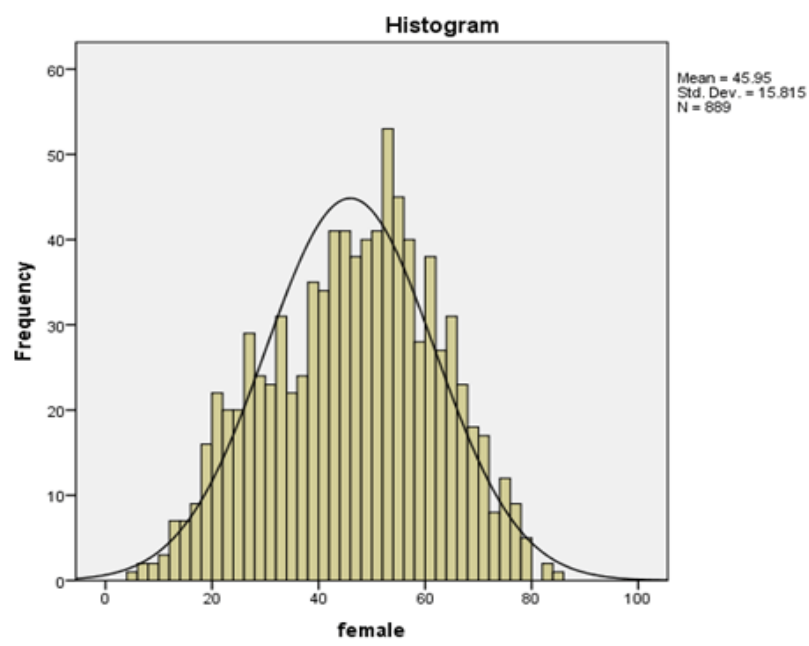

Figure 6.2: Age skewed distribution of females with cutaneous lichen planus (skewness=-0.171).

\section{Up-regulation of RIPK3 protein expression in cutaneous LP lesions}

As shown in Figure 7-1 and Figure 7-2, in normal skin tissues, RIPK3 protein was weakly expressed in the basal layer of the skin and in the bottom of the spinous layer, and it was mainly expressed in the nucleus. The positive rate in the whole epidermis was $63.33 \%$ (19 of 30 cases). In the cutaneous lichen planus, RIPK3 protein was strongly expressed in the spinous layer, basal layer and part of the granular layer, and it was mainly expressed in the cytoplasm. The positive rate was $90 \%$ (45 of 50 cases). The positive rate of the expression of RIPK3 protein in cutaneous lichen planus lesions was higher than that in normal skin tissues $(\chi 2=8.333, \mathrm{P}<0.001)$. The positive statistics of each group are shown in Table 3 and Figure 8.In the meantime, the expression intensity of RIPK3 protein in cutaneous LP was significantly higher than that in normal skin tissues $(\chi 2=29.752, \mathrm{P}<0.0001)$.

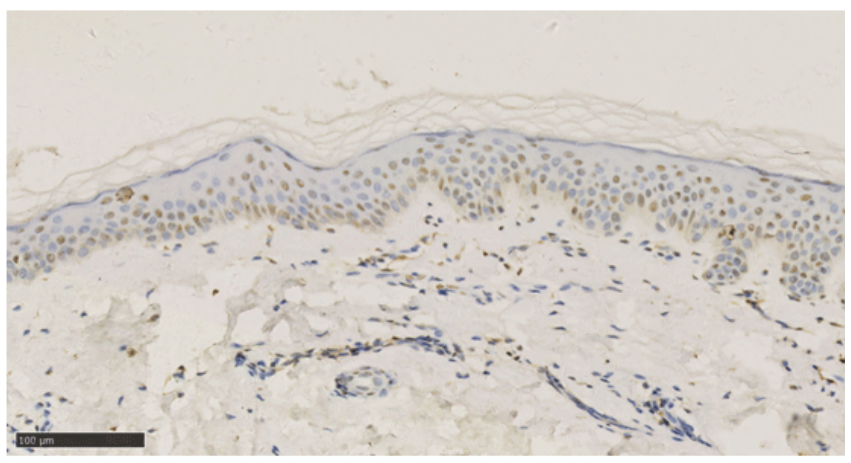

Figure 7.1: Expression of RIPK3 in normal skin (SP×200).

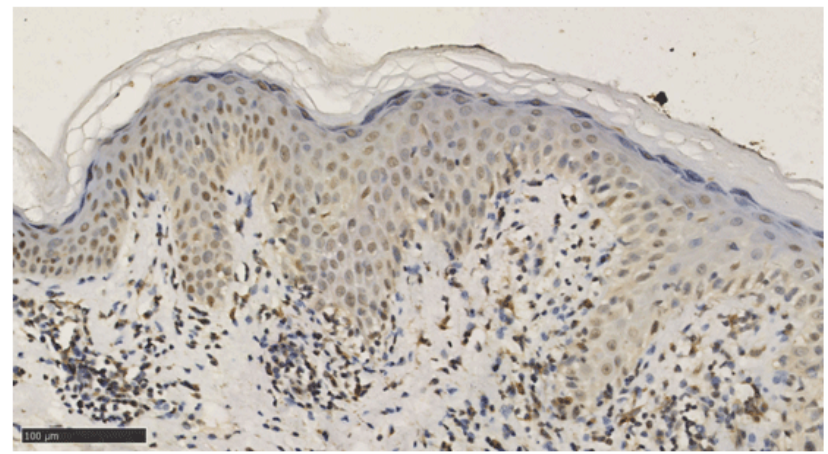

Figure 7.2: Expression of RIPK3 in cutaneous lichen planus $(\mathrm{SP} \times 200)$.

Table3: Expression of RIPK3 in normal skin and cutaneous LP.

\begin{tabular}{|c|c|c|c|c|c|c|}
\hline Groups & n & \multicolumn{4}{|c|}{ Expression grade } & Positive rate (\%) \\
\hline & & - & + & ++ & +++ & \\
\hline \begin{tabular}{c} 
Normal Skin \\
\hline $\begin{array}{c}\text { Cutaneous } \\
\text { LP }\end{array}$
\end{tabular} & 50 & 11 & 16 & 3 & 0 & 63.33 \\
\hline
\end{tabular}




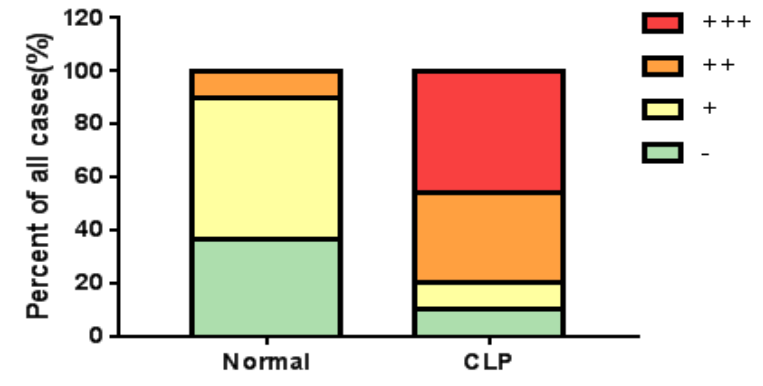

Figure 8: The statistical analysis of IHC staining for RIPK3 in cutaneous LP lesions (50 patients) orhealthy controls ( 30 subjects)

\section{Discussion}

Cutaneous lichen planus (LP) is an idiopathic inflammatory skin disease, which usually presented by purple, pruritic, polygonal, planar, papules, and plaques. The typical characteristic is Wickham striae $[1,8]$. LP can occur in various parts of the body. Lichen planus of the nails can cause thin nails, scarring, and even complete loss of the nail. Oral and vaginal lichen planus occur on the moist surfaces of the mouth and vagina, where inflammation caused by the disease often leads to surface skin layers falling off, leaving raw open areas [22-24]. Penile lichen planus can also cause erosions and scarring, but usually resembles classic lichen planus. Lichen planopilaris attacks hair follicles on the scalp leaving scarring and permanent baldness $[25,26]$. The pathologic characteristic of cutaneous LP is that the epidermis may show hyperkeratosis without parakeratosis, and apoptotic keratinocytes can be seen at a lower level. The epidermis may show as "wedge-shaped" hypergranulosis, with a characteristic "saw tooth" appearance of the rete ridges. At the dermo epidermal junction, small clefts (known as Max Joseph spaces) may be observed along with band-like lymphocytic infiltration. Pigment incontinence occurs due to the dermo epidermal junction is disrupted, which causes the increased presence of melanophages. Immunofluorescence staining of the biopsy lesions showed a bright "shaggy" band of fibrinogen along the dermoepidermal junction, as well as colloid bodies staining with any of the auto antibodies immunoglobulin M (IgM), IgG, IgA, and $\mathrm{C} 3[8,27]$.The pathogenesis of lichen planus is unclear. It may be an autoimmune disease that may be associated with cytotoxic CD8 T lymphocyte infiltration. Studies have also shown that it may be associated with overrepresentation of certain HLA haplotypes or HCV infection. Other influencing factors include drugs, environmental and psychological factors [8]. Due to the lack of good molecular targets, there is no specific target treatment for cutaneous LP, which is usually treated with topical glucocorticoids [1].

In our study, we analyzed 1854 patients' data from 2010 to 2018 in our hospital. It is proved that in the pathogenesis of lichen planus, the incidence is mostly in middle-aged and elderly people, and the mean age of onset was around 40 years old. The incidence rate was irrelevanttosex, but the age of onset is related.
The incidence of men is in earlier age, which of women is later than that of men. Making 40-year old as a demarcation point, men are more likely to onset before the point and women are more likely to onset after it. The incidence of cutaneous LP has increased in recent years.

The receptor-interacting protein kinase 3 (RIPK3), is a member of the receptor-interacting protein kinase family of serine/threonine protein kinases and contains a unique C-terminal RHIM domain that is distinct from other family members [9]. RIPK3 is considered to be a component of the TNFR1 signaling complex, which activates NF- $\kappa \mathrm{B}$ transcription factors and plays an important role in the process of necroptosis [16, $28,29]$. RIPK3 is highly expressed in lymphocytes, monocytes, and NK cells, and is also expressed in liver cells, muscle cells, retinal cells, and epithelial cells, and is abnormally expressed in inflammatory bowel disease, allergic enteritis, acute pancreatitis, Steven-Johnson syndrome, toxic epidermal necrolysis, ischemiareperfusion injury and neurodegeneration, which is implicated in the process of the disease[10-15]. In recent years, there has been ample evidence that RIPK3 plays an important role in the promotion of inflammatory diseases and abnormal proliferative skin diseases of keratinocytes [12, 13, 30,31]. To the best of our knowledge, the role of RIPK3 in cutaneous LP has not been reported. In this study, we found that expression of RIPK3 is increased in human cutaneous LP lesions and is transferred from the nucleus to the cytoplasm. This suggests that RIPK3 may play a role in the pathogenesis of cutaneous LP, and this effect is achieved by nucleoplasmic transfer.

In summary, our data demonstrate that the age of onset of males is earlier than that of females in the pathogenesis of cutaneous lichen planus, but there is no difference in sex ratios. The high incidence age of males and females is in the middle and old stage. The pathogenesis of lichen planus is very complex, and the specific mechanism has not been elucidated. We have demonstrated that the expression of RIPK3 was changed in cutaneous lichen planus and the location was transferred, which suggests that RIPK3 plays a role in the pathogenesis of cutaneous lichen planus. But the specific mechanism still needs further research.

\section{Reference}

1. Le Cleach L, Chosidow O. Lichen Planus. N Engl J Med. 2012;366:723732.

2. Patel GK, Turner RJ, Marks R. Cutaneous lichen planus and squamous cell carcinoma. European Academy of Dermatology and Venereology. 2003;17(1):97-116.

3. Madhumita Bhattacharya, Kaur I, Kumar B. Lichen Planus: A Clinical and Epidemiological Study. The Journal of Dermatology. 2000;27(8):576-582.

4. Lehman JS, Tollefson MM, Gibson LE. Lichen planus. 2009;48(7):682694.

5. Steffen C, Dupree.M. Louis-Frédéric Wickham and the Wickham's Striae of Lichen Planus. SKINmed: Dermatology for the Clinician. 2004;3(5):287-289. 
6. Pandhi D, Singal A, Bhattacharya SN. Lichen planus in childhood: a series of 316 patients. Pediatr Dermatol. 2014;31(1):59-67. doi: 10.1111/pde. 12155

7. Wagner G, Rose C, Sachse MM. Clinical variants of lichen planus. J Dtsch Dermatol Ges. 2013;11(4):309-319. doi: 10.1111/ddg.12031

8. Tziotzios C, Lee JYW, Brier T, Saito R, Hsu CK, Bhargava K, et al. Lichen planus and lichenoid dermatoses: Clinical overview and molecular basis. J Am Acad Dermatol. 2018;79(5):789-804. doi: 10.1016/j. jaad.2018.02.010

9. Pei Wen Yu, Betty CBH, Mary S, Jeff Q, Eva C, Xiang Xu, et al. Identification of RIP3, a RIP-like kinase that activates apoptosis and NFkB. Current Biology. 1999.

10. Cho YS, Challa S, Moquin D, Genga R, Ray TD, Guildford M, et al. Phosphorylation-Driven Assembly of the RIP1-RIP3 Complex Regulates Programmed Necrosis and Virus-Induced Inflammation. Cell. 2009;137(6):1112-1123. doi: 10.1016/j.cell.2009.05.037

11. Duprez L, Takahashi N, Van Hauwermeiren F, Vandendriessche B, Goossens V, Vanden Berghe T, et al. RIP Kinase-Dependent Necrosis Drives Lethal Systemic Inflammatory Response Syndrome. Immunity. 2011;35(6):908-918. doi: 10.1016/j.immuni.2011.09.020

12. Han J, Zhong CQ Zhang DW, Programmed necrosis: backup to and competitor with apoptosis in the immune system. Nature Immunology. 2011;12(12):1143-1149. doi: 10.1038/ni.2159

13. Zhao Z, Zlokovic BV, RIPped for neuroinflammation. Cell Research. 2017;27(7):849-850.

14. Oberst A, Green DR. It cuts both ways: reconciling the dual roles of caspase 8 in cell death and survival. Nature Reviews Molecular Cell Biology. 2011;12(11):757-763. doi: 10.1038/nrm3214

15. Roychowdhury S, McCullough RL, Sanz-Garcia C, Saikia P, Alkhouri $\mathrm{N}$, Matloob A et al. Receptor interacting protein 3 protects mice from high-fat diet-induced liver injury. Hepatology. 2016;64(5);1518-1533. doi: 10.1002/hep.28676

16. Moriwaki K, Chan FKM. RIP3: a molecular switch for necrosis and inflammation. Genes \& Development. 2013;27(15):1640-1649. doi: 10.1101/gad.223321.113

17. Wallach D, Kang TB, Dillon CP, Green DR. Programmed necrosis in inflammation: Toward identification of the effector molecules. Science. 2016;352(6281):aaf2154. doi: 10.1126/science.aaf2154

18. Wallach D, Kovalenko A, Kang TB. 'Necrosome'-induced inflammation: must cells die for it? Trends in Immunology. 2011;32(11):505-509. doi: 10.1016/j.it.2011.07.004

19. Lawlor KE, Khan N, Mildenhall A, Gerlic M, Croker BA, D'Cruz AA, et al. RIPK3 promotes cell death and NLRP3 inflammasome activation in the absence of MLKL. Nature Communications. 2015;6:6282. doi: $10.1038 /$ ncomms7282
20. Ciraci C, Sutterwala FS. Inhibitors of Apoptosis Proteins and IL-1 $\beta$ : A Tangled Relationship. Immunity. 2012;36(2):155-157. doi: 10.1016/j. immuni.2012.02.007

21. Moriwaki K, Balaji S, McQuade T, Malhotra N, Kang J, Chan FK. The Necroptosis Adaptor RIPK3 Promotes Injury-Induced Cytokine Expression and Tissue Repair. Immunity. 2014;41(4):567-578. doi: 10.1016/j.immuni.2014.09.016

22. Al-Hashimi I, Schifter M, Lockhart PB, Wray D, Brennan M, Migliorati $\mathrm{CA}$, et al. Oral lichen planus and oral lichenoid lesions: diagnostic and therapeutic considerations. Oral Surg Oral Med Oral Pathol Oral Radiol Endod. 2007;103 Suppl:S25 e1-12. DOI: 10.1016/j. tripleo.2006.11.001

23. Carbone M, Arduino PG, Carrozzo M, Gandolfo S, Argiolas MR, Bertolusso G, et al. Course of oral lichen planus: a retrospective study of 808 northern Italian patients. Oral Dis. 2009;15(3):235-243. doi: 10.1111/j.1601-0825.2009.01516.x

24. Bermejo-Fenoll A, Sánchez-Siles M, López-Jornet P, Camacho-Alonso F, Salazar-Sánchez N, et al. A retrospective clinicopathological study of 550 patients with oral lichen planus in south-eastern Spain. J Oral Pathol Med. 2010;39(6):491-496. doi: 10.1111/j.16000714.2010.00894.x

25. Berger,Timothy G. Lichen Planus.JAMA Dermatology. 2015;151(3):356. doi:10.1001/jamadermatol.2014.5522

26. Fox LP, Lightdale CJ, Grossman ME. Lichen planus of the esophagus: what dermatologists need to know. J Am Acad Dermatol. 2011;65(1):175-183. doi: 10.1016/j.jaad.2010.03.029

27. D W. The Lichenoid Tissue Reaction. In: Weedon's Skin Pathology. 3rd ed. Edinburgh: Elsevier Limited. 2010. 35.

28. Meylan E, Tschopp J. The RIP kinases: crucial integrators of cellular stress. Trends Biochem Sci. 2005;30(3):151-159. DOI: 10.1016/j. tibs.2005.01.003

29. Galluzzi L, Kepp O, Chan FK, Kroemer G. Necroptosis: Mechanisms and Relevance to Disease. Annual Review of Pathology: Mechanisms of Disease. 2017;12(1);103-130. DOI: 10.1146/annurevpathol-052016-100247

30. Schneider AT, Gautheron J, Feoktistova M, Roderburg C, Loosen SH, Roy S, et al. RIPK1 Suppresses a TRAF2-Dependent Pathway to Liver Cancer. Cancer Cell. 2017;31(1):94-109. doi: 10.1016/j. ccell.2016.11.009

31. Daniels BP, Snyder AG, Olsen TM, Orozco S, Oguin TH, Stephen W.G. Tait, et al. RIPK3 Restricts Viral Pathogenesis via Cell DeathIndependent Neuroinflammation. Cell. 2017;169(2):301-313.e11. doi: 10.1016/j.cell.2017.03.011 